\title{
FINITE INTERPOLATION FOR ANALYTIC FUNCTIONS WITH FINITE DIRICHLET INTEGRALS
}

\author{
MITSURU NAKAI ${ }^{1}$
}

The finite interpolation problem for AD-functions on planar (zero genus) Riemann surfaces was completely solved by Sario [2] and Rodin [1]. We shall extend their result to the case of Riemann surfaces with finite genus.

Theorem. Let $R$ be an open Riemann surface of finite genus. Given a finite number of distinct points $\zeta_{k}(k=1,2, \cdots, n)$ in $R$, local parameters $z_{k}$ at $\zeta_{k}$ with $z_{k}\left(\zeta_{k}\right)=0(k=1,2, \cdots, n)$ and complex numbers $\alpha_{\nu k}(\nu=0,1, \cdots, m ; k=1,2, \cdots, n)$. Then there exists a bounded analytic function $f$ with finite Dirichlet integral on $R$ such that

$$
\frac{d^{v} f}{d z_{k}^{v}}\left(\zeta_{k}\right)=\alpha_{\nu k} \quad(\nu=0,1, \cdots, m ; k=1,2, \cdots, n)
$$

if and only if $R$ does not belong to the class $0_{\mathrm{AD}}$.

Proof. The necessity of the condition $R \notin 0_{\mathrm{AD}}$ is evident. We have to show the solvability of (1) under the condition $R \notin 0_{\mathrm{AD}}$. Since $R$ has finite genus, $R \notin 0_{\mathrm{AD}}$ implies the existence of a nonconstant ABDfunction $F(z)$ on $R$. Let $R^{*}$ be a closed Riemann surface which contains $R$ as a subsurface. Choose a point $\zeta_{0}$ in $R-\left\{\zeta_{1}, \zeta_{2}, \cdots, \zeta_{n}\right\}$ such that $F\left(\zeta_{0}\right) \neq F\left(\zeta_{k}\right)(k=1,2, \cdots, n)$. For each fixed $k(k=1,2, \cdots, n)$, by Riemann-Roch's theorem, there exists a meromorphic function $r_{k}(z)$ on $R^{*}$ such that $r_{k}(z)$ has a simple pole at $\zeta_{k}$ and a pole of order $n_{k}$ at $\zeta_{0}$ and regular on $R^{*}-\left\{\zeta_{0}, \zeta_{k}\right\}$. Let $m_{k}$ be the order of zero of the function $\prod_{j=1}^{n}\left(F(z)-F\left(\zeta_{j}\right)\right)^{m+1}$ at $\zeta_{k}$ and let $s=\max \left\{m_{k} n_{k} ; k=1,2, \cdots, n\right\}$. Put

$$
H(z)=\left(F(z)-F\left(\zeta_{0}\right)\right)^{s} \prod_{j=1}^{n}\left(F(z)-F\left(\zeta_{j}\right)\right)^{m+1},
$$

which belongs to the class $\operatorname{ABD}(R)$. By construction, $\left(d^{m_{k}} H / d z_{\mathfrak{k}}^{m_{k}}\right)\left(\zeta_{k}\right)$ $\neq 0$ and $\left(z_{k} r_{k}\right)\left(\zeta_{k}\right) \neq 0$. Hence for each $\nu(\nu=0,1, \cdots, m)$, we may set

$$
H_{\nu k}(z)=\left[(\nu !) \cdot \frac{1}{m_{k} !} \cdot \frac{d^{m_{k}} H}{d z_{k}^{m_{k}}}\left(\zeta_{k}\right)\left(\left(z_{k} r_{k}\right)\left(\zeta_{k}\right)\right)^{m_{k}-\nu}\right]^{-1} \cdot\left(r_{k}(z)\right)^{m_{k}-\nu} \cdot H(z) .
$$

Received by the editors August 18, 1965.

1 This work was sponsored by the U. S. Army Research Office, Durham, Grant DA-AROD-31-124-G499, University of California, Los Angeles. 
Again from the construction it is easy to see that $H_{v k}$ belongs to $\operatorname{ABD}(R)$ and for each $k(k=1,2, \cdots, n)$,

$$
H_{0 k}\left(\zeta_{j}\right)=\delta_{k j} \quad(j=1,2, \cdots, n)
$$

and moreover for each fixed $\nu(\nu=1,2, \cdots, m)$,

$$
\begin{aligned}
& \frac{d^{\mu} H_{\nu k}}{d z_{j}^{\mu}}\left(\zeta_{j}\right)=0 \quad(\mu=0,1, \cdots, \nu-1 ; j=1,2, \cdots, n), \\
& \frac{d^{\nu} H_{\nu k}}{d z_{j}^{\nu}}\left(\zeta_{j}\right)=\delta_{k j} \quad(j=1,2, \cdots, n) .
\end{aligned}
$$

Define $m+1$ functions $P_{\nu}(z)(\nu=0, \cdots, m)$ on $R$ inductively by

$$
P_{\nu}(z)=P_{\nu-1}(z)+\sum_{j=1}^{n}\left(\alpha_{\nu j}-\frac{d^{\nu} P_{\nu-1}}{d z_{j}^{\nu}}\left(\zeta_{j}\right)\right) H_{\nu j}(z) \quad(\nu=0, \cdots, m)
$$

with $P_{-1}=0$. Then $f(z)=P_{m}(z)$ belongs to $\operatorname{ABD}(R)$ and satisfies (1).

COROLlary. Let $R$ be an open Riemann surface of finite genus not belonging to the class $0_{\mathrm{AD}}$ and $\mathcal{F}=\mathcal{F}\left(\left(\zeta_{k}\right),\left(z_{k}\right),\left(\alpha_{v k}\right)\right)$ be the class of all AD-functions $f$ on $R$ satisfying the interpolating condition (1). Then the class $\mathcal{F}$ is not empty and there exists a unique function $f_{0}$ in $\mathcal{F}$ such that

$$
D(f)=D\left(f_{0}\right)+D\left(f-f_{0}\right)
$$

for any $f$ in $\mathcal{F}$ and a fortiori $f_{0}$ is the unique solution with minimum norm of the interpolation problem given by (1):

$$
D\left(f_{0}\right)=\min \{D(f) ; f \in \mathfrak{F}\} .
$$

Proof. For each closed parametric disk $K_{k}$ with local parameter $z_{k}(k=1,2, \cdots, n)$ and for any relatively compact parametric disk $U$ with local parameter $z$ such that $\zeta_{k} \in U(k=1,2, \cdots, n)$, by the local subharmonicity of $\left|f^{\prime}\right|^{2}$ for $f \in \mathcal{F}$ and Cauchy's inequalities, we can find a constant $c_{U}$ such that

(2) $\left|f_{1}(z)-f_{2}(z)\right|^{2}+\sum_{k=1}^{n} \sum_{\nu=0}^{m}\left|\frac{d^{v} f_{1}}{d z_{k}^{\nu}}\left(z_{k}\right)-\frac{d^{\nu} f_{2}}{d z_{k}^{\nu}}\left(z_{k}\right)\right|^{2} \leqq c_{U} D\left(f_{1}-f_{2}\right)$

for any $z \in U, z_{k} \in K_{k}(k=1,2, \cdots, n)$ and $f_{1}, f_{2} \in F$. Let $\left\{f_{n}\right\}$ be a sequence such that $\left\{f_{n}\right\} \subset \mathcal{F}$ and $\lim _{n} D\left(f_{n}\right)=d=\inf \{D(f) ; f \in \mathcal{F}\}$. Since $\left(f_{n}+f_{n+p}\right) / 2 \in \mathcal{F}$ and

$$
\begin{aligned}
D\left(f_{n}-f_{n+p}\right) & =2\left(D\left(f_{n}\right)+D\left(f_{n+p}\right)\right)-4 D\left(\frac{f_{n}+f_{n+p}}{2}\right) \\
& \leqq 2\left(D\left(f_{n}\right)+D\left(f_{n+p}\right)\right)-4 d,
\end{aligned}
$$


we conclude that $\lim _{n} D\left(f_{n}-f_{n+p}\right)=0$ for any $p$. This with (2) gives the existence of a function $f_{0}$ in $\mathcal{F}$ such that $\lim _{n} D\left(f_{n}-f_{0}\right)=0$ so that $D\left(f_{0}\right)=d$. For any $f \in \mathcal{F}$ and any complex number $\lambda, f_{0}+\lambda\left(f-f_{0}\right)$ $\in \mathcal{F}$. Hence $D\left(f_{0}+\lambda\left(f-f_{0}\right)\right) \geqq D\left(f_{0}\right)$. Whence it follows that $D\left(f_{0}, f-f_{0}\right)=0$. Therefore $D(f)=D\left(f_{0}+\left(f-f_{0}\right)\right)=D\left(f_{0}\right)+D\left(f-f_{0}\right)$ $=d+D\left(f-f_{0}\right)$. Thus $D(f)=d$ if and only if $f=f_{0}$.

\section{REFERENCES}

1. B. Rodin, Reproducing formulas on Riemann surfaces, Doctoral Dissertation, University of California, Los Angeles, Calif., 1961.

2. L. Sario, Extremal problems and harmonic interpolation on open Riemann surfaces, Trans. Amer. Math. Soc. 79 (1955), 362-377.

University of California, Los Angeles and NAGOYA UNIVERSITY 